\section{Asthma: Ungenügende Versorgung beim Hausarzt}

\section{Die Empfehlungen zur Asthmatherapie werden von deutschen Hausärzten immer noch unzureichend umgesetzt, so das Fazit einer neuen Untersuchung. Verbesserungswürdig sei sowohl das Wissen um die antientzündliche Basistherapie wie auch das Verhalten bei akuten Exazerbationen und der Umgang mit Peak- flow-Metern bzw. mit den Inhalationssystemen.}

Ziele der Asthmatherapie sind die Kontrolle von Symptomen wie Atemnot oder Husten mit Erhaltung einer möglichst hohen Lebensqualität sowie langfristig die Prophylaxe von Asthmaanfällen und das Verhindern von Komplikationen wie Lungenemphysem und respiratorische Insuffizienz.

In der medikamentösen Therapie sind sich internationale wie nationale Expertengremien einig: Basis bilden die inhalativen Kortikoide - auch bei den leichteren Erkrankungsformen - inhalative Broncholytika sollen nur noch kurzfristig bei Bedarf eingesetzt werden. Orale Asthmamedikamente kommen nur dann in Frage, wenn mit hochdosierten inhalativen Steroiden keine ausreichende Wirkung mehr erreicht wird.

\section{Wissen teilweise mangelhaft}

Obwohl diese Richtlinien - zuletzt auf nationaler Ebene von der Deutschen Atemwegsliga 1998 aktualisiert - zumindest theoretisch den meisten Ärzten bekannt sind, scheinen die Empfehlungen gerade bei den Hausärzten immer noch nicht optimal umgesetzt zu werden. Dies ergab jetzt erneut eine Untersuchung im Rahmen des Drug Education Project, ein von der EU unterstütztes internationales Projekt mit dem Ziel der Förderung einer rationalen Pharmakotherapie durch Ärztefortbildung in Kleingruppen.

Noch vor Beginn der Fortbildungsveranstaltungen wurden Hausärzte (Fachärzte für Allgemeinmedizin, praktische Ärzte und niedergelassene hausärztlich tätige Ärzte ohne weitere Gebietsbezeichnung) in $7 \mathrm{KV}$-Bezirken im Umkreis von Göttingen zur Teilnahme an ei-

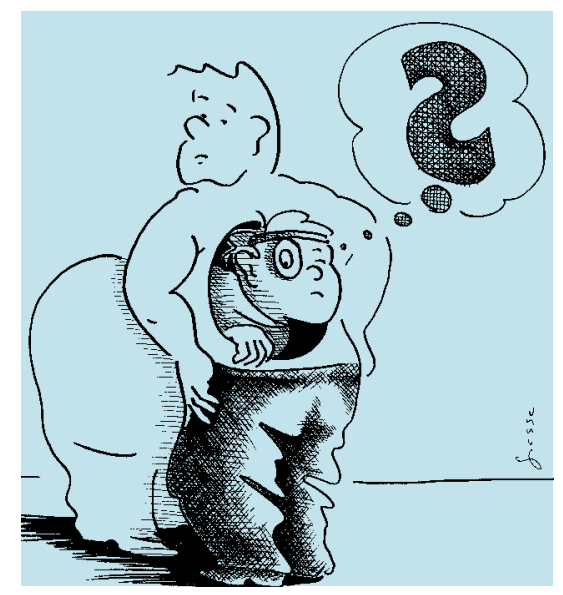

ner Fragebogenaktion gebeten. 76 Hausärzte kamen dieser Bitte nach und beantworteten Fragen über die Asthmaerkrankung und deren Therapie, 55 von ihnen gewährten darüber hinaus Einsicht in ihre Arzneimittelverordnungen der letzten 5 Monate.

Die Analyse der Fragebogenaktion ergab, dass der überwiegenden Mehrheit der Hausärzte (83\%) die Empfehlungen der Deutschen Atemwegsliga bekannt waren, 65\% stimmten mit den Therapievorschlägen überein. Insgesamt wurden immerhin 69\% der Fragen richtig beantwortet, wobei der Wissensstand zur Überwachung und Erhaltungstherapie noch am besten war. Allerdings vertraten $22 \%$ der Befragten noch die Meinung, dass inhalative Kortikoide erst so spät wie möglich eingesetzt werden dürften und 19\%, dass bei Nachlassen der Atembeschwerden diese Basismedikamente abzusetzen seinen.

Schlechter sah es bei der Bewältigung akuter Exazerbationen aus. Gelb- grünes Sputum sei hier ein Hinweis auf einen bakteriellen Infekt - 86\% der Ärzte bejahten die Frage fälschlicherweise. $21 \%$ halten in einem solchen Fall orale Steroide für kontraindiziert. Und 74\% würden einem Asthmatiker, der unter zunehmender Obstruktion mit Sputumauswurf leidet, ein Antibiotikum verordnen, 58\% stimmten der Aussage zu, dass ein orales Kortikoid indiziert sei.

Schlusslicht bildete der Kenntnisstand zur Handhabung von Pulverinhalatoren bzw. Dosieraerosolen. sowie der Gebrauch von Peak-flow-Metern.

\section{Vernachlässigte Basismedikation}

Für die Auswertung der Verordnungsprofile von 55 Ärzten konnten 290 Asthmapatienten mit 1020 Verordnungen relevanter Medikamente in den Datensätzen der AOK Niedersachsen identifiziert werden. Es zeigte sich, dass viele Patienten keine sinnvolle Dauertherapie erhielten. Oft wurden zwar hohe Dosen inhalativer Broncholytika, aber keine Steroide bzw. Cromoglicinsäure in ausreichend hoher Dosierung verschrieben.

Die Empfehlung, akute Verschlechterungen antientzündlich statt antibiotisch zu behandeln, wird von Hausärzten kaum umgesetzt. Viele Patienten erhalten dagegen ständig oder intermittierend Hustenmittel - deren Effektivität ist aber bei asthmabedingtem Husten umstritten. Besonders bedenklich: Rund $10 \%$ der Patienten erhalten hustendämpfende Präparate, oft sogar gleichzeitig mit auswurffördernden Mitteln.

\section{Fortbildung dringend erforderlich}

Auch wenn die vergleichsweise geringe Zahl von Asthmapatienten in dieser Erhebung noch keine Rückschlüsse auf die Versorgungsqualität in Deutschland zulässt, kann doch vermutet werden, dass der Kenntisstand vieler Hausärzte zur Behandlung des Asthma verbessert werden muß - so das Fazit der Autoren. Praktische Fertigkeiten wie auch das Wissen um antientzündliche Medikamente sollten in Fortbildungen noch intensiver als bisher vermittelt werden. $b k$

\section{Hummers-Pradier E, Kochen MM}

Hausärztliches Vorgehen bei Patienten mit Asthma bronchiale.

Z Allg Med 1999; 75: 375-8o. 